\title{
Front-of-pack warning labels are preferred by parents with low education level in four Latin American countries
}

Sofía Rincón Gallardo Patiño*1 ${ }^{*}$ Ángela Carriedo ${ }^{2}$, Lizbeth Tolentino-Mayo ${ }^{3}$, Jacqueline Araneda $^{4}$, Lorena Allemandi ${ }^{5}$, Adriana Murillo ${ }^{6}$, and Simon Barquera ${ }^{7}$

1 Department of Human Nutrition, Foods, and Exercise; College of Agriculture and Life Sciences; Virginia Polytechnic Institute and State University, Blacksburg, VA, USA; sofiargp@vt.edu

2 World Public Health Nutrition Association; London, United Kingdom; angela_carriedo@yahoo.com

3 Centro de Investigación en Nutrición y Salud; Instituto Nacional de Salud Pública, Cuernavaca, Morelos, México; mltolentino@insp.mx

4 Departamento de Nutrición y Salud Pública; Universidad de Bío-Bío, Chillán, Chile; jaraneda@ubiobio.cl

5 Políticas de Alimentación Saludable, Fundación Interamericana del Corazón, Argentina; lorena.allemandi@ficargentina.org

6 Escuela de Nutrición; Universidad de Costa Rica; San José, San Pedro, Costa Rica; ADRIANA.MURILLO@ucr.ac.cr

${ }^{7}$ Centro de Investigación en Nutrición y Salud; Instituto Nacional de Salud Pública, Cuernavaca, Morelos, México; sbarquera@insp.mx

* Correspondence: sofiargp@vt.edu; Tel.: +1-540-831-9719 


\section{Abstract}

Background: Overweight and obesity rates in Latin America are much higher than the global prevalence. Front-of-pack (FOP) nutrition label systems are gaining momentum in Latin America and being implemented as a strategy to tackle obesity among children and adults. This study investigates the usage and preference of three FOP nutrition labels among Argentinian, Chilean, Costa Rican, and Mexican adult parents.

Methods: From March to May 2016, a questionnaire was administered to 966 parents of elementary school aged children in Argentina (89), Chile (304), Costa Rica (258) and Mexico (315). Guideline Daily Amounts (GDA), traffic light (TL), and warning label FOP systems were compared and analyzed. Statistical analysis was conducted using frequencies and proportions. Median differences tests, ANOVAs and logistic regression models were performed. A thematic analysis of qualitative data was conducted using a deductive process.

Results: Parents with low education levels and poor health conditions (i.e., overweight) preferred the FOP warning labels over the GDA and TL systems. The GDA FOP nutrition labels were preferred by parents with higher education levels. Overall, the TL system was preferred with no significant differences across countries.

Conclusion: FOP warning labels are preferred by parents with vulnerable conditions (i.e., low education levels and overweight) across four Latin American countries. An easily understandable, readable and acceptable label may facilitate usage by vulnerable groups to make healthy food purchases. These results have important policy implications by providing evidence to government policymakers to enact legislation to implement or enhance existing FOP nutrition label policies as a viable strategy to reduce obesity risk.

Key words: Front-of-Pack, nutrition, label, policy, obesity, Latin America 


\section{Introduction}

Poor quality diets of children and adults have contributed to a rapid increase of obesity and dietrelated non-communicable diseases (NCDs) worldwide and are considered a major public health problem (Ezzati and Riboli 2013). In 2016, the World Health Organization estimated that more than 1.9 billion (39\%) of the adult population was overweight, 650 million (13\%), obese, and 340 million of children 5 - 19 years old were overweight or obese (World Health Organization 2018). However, in Latin America, the rates are much higher with 302 million (57\%) adults overweight and 100.8 million (19\%) obese (Ng, et al. 2014). A study from Rivera et al. (2014) estimated that 3.8 million children younger than 5 years, 22.2-25.9 million school-age children, and 16.5-21.1 million adolescents were overweight or obese in the region. In this region, obesity has increased more rapidly among the most vulnerable populations (i.e., low socioeconomic groups) (Jiwani et al. 2019).

The increasing rates of obesity throughout this region have been associated with the nutrition transition currently taking place in which dietary patterns have shifted toward energy-dense foods and beverages that are high in fat, sugar, and salt, while low in other essential nutrients (Popkin and Reardon 2018). Eating behaviors are one of the main causes of the obesity epidemic and highly influenced by exposure and access to unhealthy food and beverage products. Latin America represents $16.8 \%$ of the ultra-processed market share globally followed by North America and Asia Pacific (Pan American Health Organization 2019). These foods have a high caloric content and high levels of sugar, salt and fat. In our evolving society, where lifestyles have decreased time available to prepare and consume homemade food, purchases of foods (including ready-to make and ultra-processed foods, sales of which increased almost 40\% from 2000-2013 in LA) now represent a significant portion of the total energy intake daily

(Drewnowski and Rehm 2013; Poti et al 2014; Pan American Health Organization 2019).

As policymakers of the household, parents have normally been held responsible for making household food purchasing and feeding decisions for their children (De la Cruz-Gongora et al. 2012). Hence, parents’ purchases influence the family diet, especially children's food and beverage intake. Parents' willingness to provide healthy options to their families and children is constantly jeopardized by factors that influence food and beverage product purchases such as time, price, and lack of information or skills to interpret the nutrition labels. Additionally, the commercial food and beverage manufacturers have engineered ways to attract vulnerable populations, like worried parents, to keep consuming those foods by extensively targeting them with promotional messages. They do this not only with targeted advertising, but with attractive packaging, exploiting a series of existing misleading FOPL systems, such as health and nutrition claims (Ronit and Jensen 2014).

Therefore, robust and evidence-based regulatory measures such as mandatory Front-of-Pack (FOP) nutrition labels systems have been implemented in in some countries in Latin America and worldwide, and are considered to be a cost-effective strategy to improve diets, and therefore impacting on obesity and diet-related NCDs (World Health Organization 2013; Kline et al. 2017). A robust body of evidence demonstrates that clear and simple FOP nutrition label systems help consumers to identify healthier foods and beverages among an extensive variety of products, allowing consumers to improve their diets towards healthier options (Shangguan et al. 2019; Anastasiou et al 2019; Cowburn and Stockley 2005; Campos et al 2011; Talati et al. 2019). 
The Food and Agricultural Organization defines FOP nutrition label as "any written, printed or graphic matter that is present on the front label of packaged foods and beverages." (Food and Agricultural Organization 2019) By 2016, the time when the present research was conducted, three different types of FOP nutrition labels were formerly approved by Latin American governments (as illustrated in Figure 1). The Guideline Daily Amounts (GDA) is considered a nutrient-specific label that displays product content information based on the average daily amount of energy and percentages of nutrients (fats, sugar, sodium and energy) per serving (World Health Organization 2019b). Another nutrient-specific system is the Traffic Light (TL) with a color-coded format that carry information on the content of fat, sugar, and salt represented by one of the three traffic light colors (i.e., high-red, medium-yellow and low-green). The FOP warning labels (WL) indicates aspects of the product that trigger warnings to the consumer about an excess of an ingredient that has been associated with adverse health consequences (World Health Organization 2019b).

Figure 1. Types of Front-of-pack nutrition labels assessed in Argentina, Chile, Costa Rica, and Mexico

a. Guideline Daily Amounts

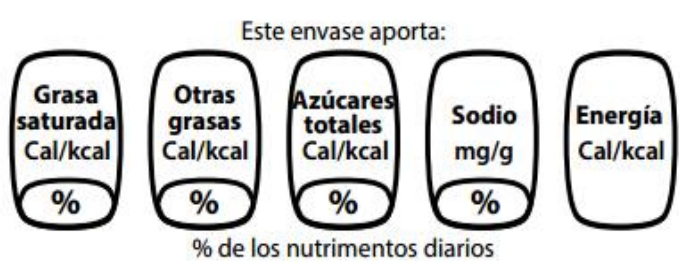

b. Traffic Light

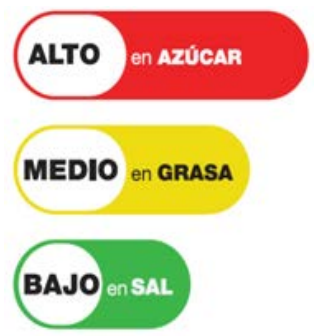

c. Warning labels

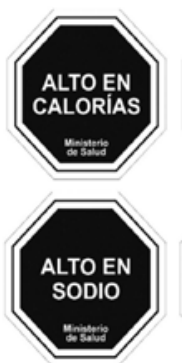

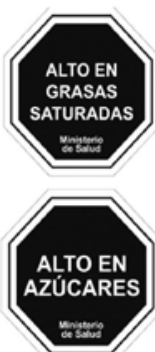

In 2014, the Ecuadorian government implemented a mandatory policy to use the FOP TL, also used by the United Kingdom (Ministry of Public Health Ecuador 2013). During the same year, Mexico introduced the GDA system as mandatory (Ministry of Health Mexico 2014). In June 2016, Chile mandated all packaged food and beverages with high content of saturated fats, sugar, or sodium to display a FOP WL (Ministry of Health Chile 2015). Since then, a growing number of countries have been implementing the WL system, including Peru (Michail 2019b) and Uruguay (Graña 2018). Most recently, in 2019, the Mexican government approved a law to replace the GDA with WLs (Sherman 2019). Other countries in the Latin American region have developed recommendations to the government (i.e., Brazil) (Khandpur et al. 2018) or are having discussions in their congresses (i.e., Argentina and Colombia) to implement the WL system.

Given that various FOP nutrition label systems are in place in Latin America, and that more countries are considering implementing this strategy to tackle obesity, it is important to understand which FOP nutrition labels consumers prefer, since this might increase usage. It is especially important to understand parents' views, whose role in their childrens' diets is essential. It is well documented that overweight and obese children are more likely to develop other NCDs at an early stage of life (World Health Organization 2019a). 
To the best of our knowledge, there are no studies to date that evaluate parental preferences for and usage of the FOP nutrition label systems currently in use across the Latin American region. Therefore, this article aims to assess the usage and preference of FOP nutrition labeling systems with a purposive sample of parents of elementary school-aged children in four countries of Latin America (Argentina, Chile, Costa Rica, and Mexico).

\section{Methods}

This is a mixed method study using a questionnaire with closed and open questions to a purposive sample of parents of elementary school children in four countries in Latin America. From March to May 2016, the questionnaire was administered to parents in Argentina, Chile, Costa Rica, and Mexico. The GDA, TL, and WL FOP labeling systems (Figure 1) were compared and analyzed.

\section{Questionnaire}

A 27-item questionnaire with 25 close-ended questions and 2 open-ended questions was used to collect data on the usage, perceptions, and preferences regarding FOP nutrition labels in terms of legibility, clarity, and nutrition. The questionnaire was adapted from one used and validated in Mexico. The design and application of some of the questions included have been published elsewhere (Stern, et al. 2011; Shamah-Levy 2016).

The questionnaire included three sections. Section I consisted of demographic characteristics of participants (i.e., gender, age, body weight and height, employment, education level achieved, and household with special diet). Each question had multiple-choice answers except for body weight and height, which were self-reported. Section II evaluated usage and III preferences regarding FOP using a 5 -point Likert scale " $1=$ never to $5=$ always" and " $1=$ totally disagree to $5=$ totally agree”, respectively. The open-ended questions explored reasons for specific preferences of participant responses.

The adapted questionnaire was piloted twice: first, to test the feasibility of the instrument with research experts on the topic; second, to test the acceptability with a subsample of the targeted population in each country. Then, the questionnaire was adapted with appropriate terms and language used in each country.

\section{Sampling}

Argentina, Chile, Costa Rica, and Mexico were selected on the basis of geographical distribution (North, Central, and South of Latin America) and willingness to participate in the study.

First, we stratified elementary schools by public and private sectors. Then, a convenience sample of elementary schools in each country was selected. We included elementary schools from public and private sectors, rural and urban, that had at least 200 students and were lay or non-religious. Schools were excluded if attendance was single-gendered, if the permission to do our study was 
rejected, or if the school was participating in another research project. Participants were parents with at least one child enrolled in the selected elementary school; parents employed as health professionals or working in the food industry were excluded.

\section{Data collection}

Prior to data collection, a written consent form was obtained from each parent that decided to participate. The research protocol, questionnaire and consent form were approved by the National Institute of Public Health of Mexico, the University of Chile's Institute of Nutrition and Food Technology (INTA), the University of Costa Rica, and the InterAmerican Heart Foundation Argentina (FIC).

Questionnaires were distributed to parents that met the inclusion criteria, and in the presence of the fieldwork staff, they were given time to self-respond. The questionnaire took an average of 10 minutes to be completed. In Mexico, all four countries' data were coded by two trained and standardized persons into an excel database file, and open questions into an NVivo software's file Inter-coder. To diminish coding errors, data were entered twice.

\section{Data analysis}

Statistical and thematic analyses were performed. The reliability or internal consistency of the questionnaire was measured using Cronbach's Alpha. The alpha coefficient of reliability for the four sections in the questionnaire suggests a high internal consistency (92.7\%).

Descriptive analyses were conducted using frequencies and proportions. Usage of FOP labels by the parental respondents was assessed by median differences test and ANOVAs. Logistic regression models were performed to identify if sociodemographic characteristics modified the effect of FOP nutrition label system preference. Statistical significance was defined by $\mathrm{p}<0.05$. All statistical analyses were performed using Stata software version 14.0.

The thematic analysis was conducted using a deductive process. First, we generated initial codes, followed by searching themes among codes, and producing final categories. A codebook was developed with the main categories, themes, and codes to complete the analysis. To increase readiness of the qualitative data, a matrix was used to display results. The accuracy of the analysis was confirmed by a triangulation process (Massey 1999; Carter et al. 2014), where two researchers coded and analyzed the data independently and followed joint consensus to solve discrepancies.

\section{Results}

Table 1 shows the sociodemographic characteristics of the parents that participated in the study. A total of 966 parents completed the survey: 89 Argentina, 304 Chile, 258 Costa Rica and 315 Mexico. We excluded an additional 27 questionnaires from participants with missing data. 
Table 1. Descriptive characteristics of participants*, stratified by country

\begin{tabular}{|c|c|c|c|c|c|}
\hline & Argentina & Chile & Costa Rica & Mexico & Total \\
\hline Sample (n) & 89 & 304 & 258 & 315 & 966 \\
\hline \multicolumn{6}{|l|}{ Gender (\%) } \\
\hline Female & 74.2 & 73.6 & 83.3 & 84.1 & 79.7 \\
\hline Male & 25.8 & 26.3 & 16.7 & 15.9 & 20.3 \\
\hline Age (years; mean \pm SD) & $38.2 \pm 7.7$ & $38.7 \pm 6.0$ & $38.4 \pm 8.9$ & $36.3 \pm 7.8$ & $37.8 \pm 7.6$ \\
\hline \multicolumn{6}{|l|}{ Educational level reached (\%) } \\
\hline None & 1.0 & 1.6 & 9.3 & 1.3 & 3.4 \\
\hline Elementary School & 3.9 & 9.1 & 26.6 & 5.7 & 12.1 \\
\hline Middle School & 23.5 & 5.4 & 20.8 & 15.6 & 14.5 \\
\hline High school/Technical & 59.8 & 42.9 & 20.1 & 47.9 & 40.3 \\
\hline Bachelor’s degree & 11.8 & 31.2 & 17.0 & 24.8 & 23.5 \\
\hline Graduate degree & 0.0 & 9.8 & 6.2 & 4.8 & 6.2 \\
\hline \multicolumn{6}{|l|}{ Civil status (\%) } \\
\hline Single & 29.2 & 30.6 & 31.8 & 30.2 & 30.6 \\
\hline Married & 58.4 & 63.2 & 57.4 & 59.0 & 59.8 \\
\hline Divorced & 11.2 & 5.6 & 9.7 & 8.9 & 8.3 \\
\hline Widow & 1.1 & 0.6 & 1.2 & 1.9 & 1.2 \\
\hline \multicolumn{6}{|l|}{ Employment (\%) } \\
\hline Employed & 91.0 & 66.8 & 29.8 & 70.2 & 60.3 \\
\hline Unemployed & 5.6 & 28.6 & 42.3 & 27.6 & 29.8 \\
\hline Student & 1.2 & 3.9 & 5.4 & 2.2 & 3.5 \\
\hline Other & 2.2 & 0.7 & $22.5^{* *}$ & 0.0 & 6.4 \\
\hline \multicolumn{6}{|l|}{ School (\%) } \\
\hline $\begin{array}{l}\text { Public } \\
\end{array}$ & 51.7 & 45.7 & 70.2 & 62.5 & 58.3 \\
\hline Private & 48.3 & 54.3 & 29.8 & 37.5 & 41.7 \\
\hline
\end{tabular}

*Participants were parents of elementary school-aged children from Argentina, Chile, Costa Rica and Mexico (n= 966) that completed a questionnaire carried out at public and private elementary schools between March and May 2016. **Most of the Costa Ricans respondents were housewives.

\section{Usage}

In all the countries, more than one-quarter of the participants responded that they almost always (16.8\%) or always (15.7\%) use the FOP label at the moment of purchasing food or beverage products for their children (Figure 2). There were significant differences between countries $(p<0.001)$ Chilean parents $(19.4 \%)$ reported always using the FOP label at the moment of purchasing food or beverage products for their children, 14.9 percent more than Argentineans (4.5\%), and 13.4 percent more than Mexicans (6\%) $(p<0.001)$. Among Costa Rican respondents, $27 \%$ reported always using the FOP label, 21.1 percent more than Mexicans $(p<0.05)$.

There were significant differences ( $p=0.023$ ) when stratifying by level of education. Results show that the usage of FOP nutrition labels of parents at the moment of buying a food or beverage product to their children is higher with a higher level of education (Figure 3). 
Figure 2. Usage of the FOP* nutrition labels by parents from Latin America when purchasing food and beverage products for their children, by country.

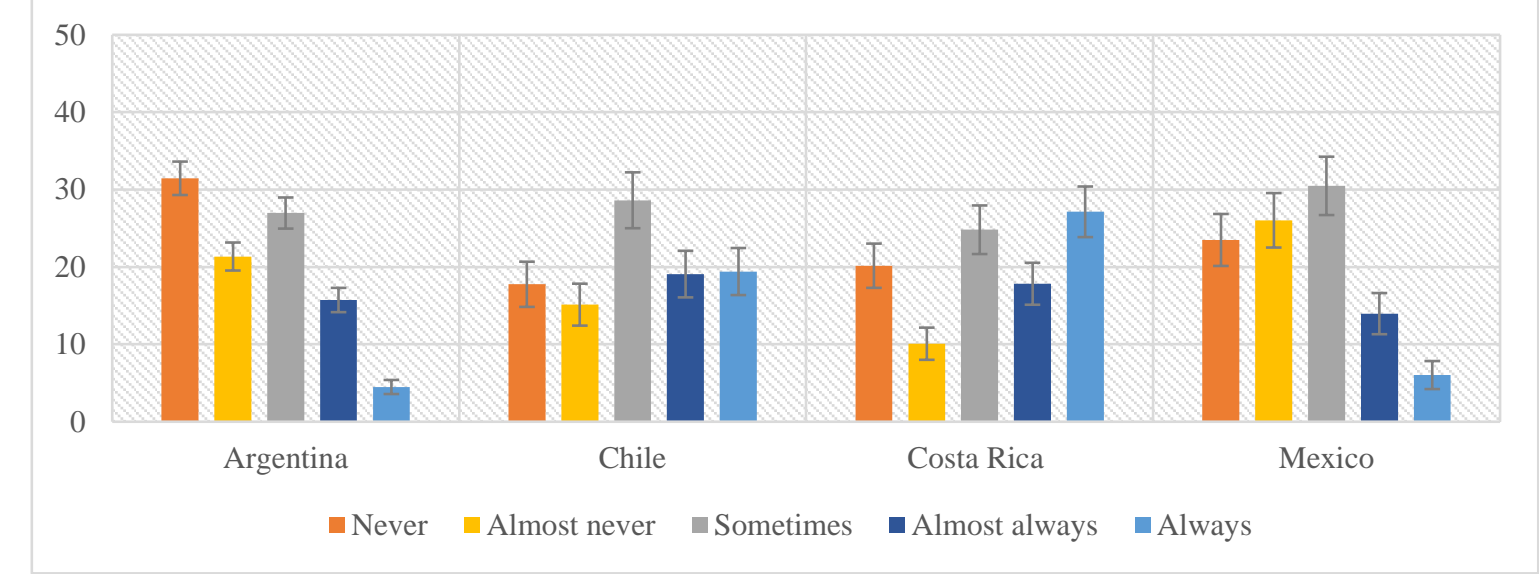

*Front-of-Pack (FOP) nutrition label use by country, questionnaire among parents of elementary school-aged children from Argentina, Chile, Costa Rica and Mexico (n= 966). Values are means with their $95 \%$ confidence intervals represented by vertical bars. The Y axis represent percentage of parents that use FOP nutrition labels when purchasing food and beverage products for their children.

Figure 3. Usage of the FOP* nutrition labels by parents from Latin America when purchasing food and beverage products for their children, by education level.

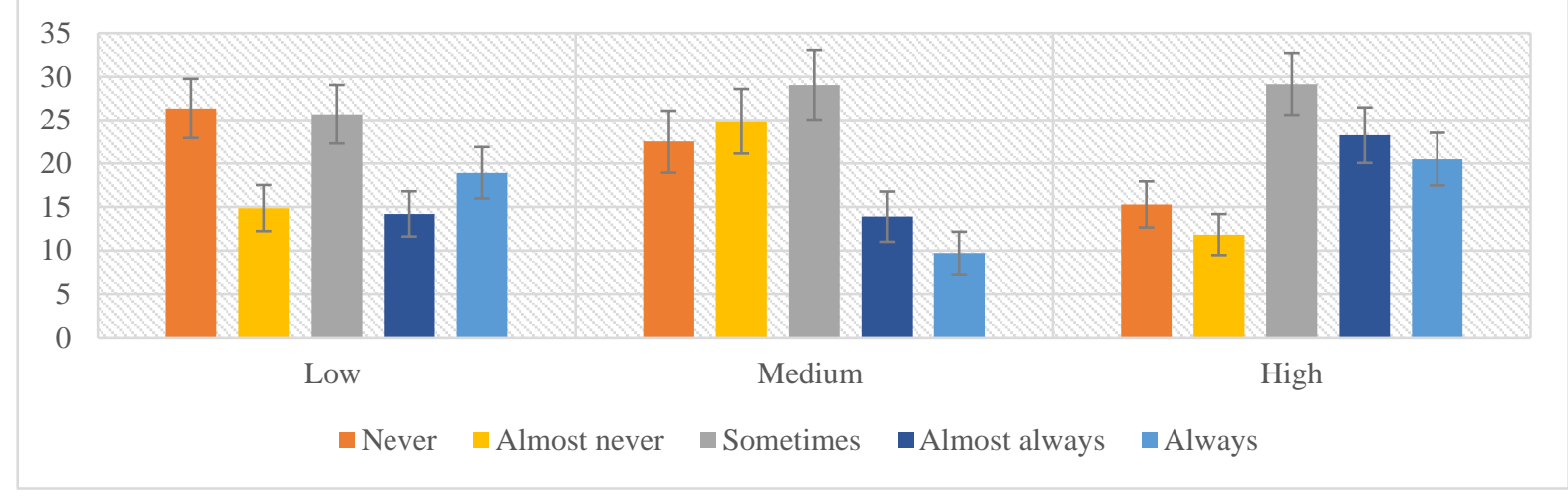

*Front-of-Pack (FOP) nutrition label use by education level, questionnaire among parents of elementary school-aged children from Argentina, Chile, Costa Rica and Mexico (n= 966). Values are means with their 95\% confidence intervals represented by vertical bars; Education level: Low- elementary school; medium - middle-school, high-school or technical career; high- bachelor's or graduate degree. The Y axis represent percentage of parents that use FOP nutrition labels when purchasing food and beverage products for their children.

\section{Preference}

A logistic model was fitted to the data to identify associations between the likelihood to prefer a FOP nutrition label and sociodemographic characteristic of the participants (Table 2). The variables that did not contribute significantly to the model were excluded (e.g., civil and employment status).

According to the model, parents with high education level were 2.47 times (95\%CI: 1.55-3.95) more likely to prefer the GDA system compared to respondents with low education level. Respondents with medium education level were 1.76 times (95\% CI: 1.13-2.74) more likely to prefer the GDA system compared to respondents with low education level; given that the 
reference group (coded $=0$ ) was low education level. No statistically significance results were found for preference towards the TL. In contrast, the odds to prefer the WL system were 2.22 times higher (95\% CI: 1.46-3.36) higher among overweight parents.

Table 2. Associations between the likelihood to prefer a FOP nutrition label and sociodemographic characteristic among Latin American parents from four countries.

\begin{tabular}{|c|c|c|c|c|c|c|}
\hline & \multicolumn{2}{|c|}{ Guideline Daily Amounts } & \multicolumn{2}{|c|}{ Traffic Light } & \multicolumn{2}{|c|}{ Warning labels } \\
\hline & OR (CI 95\%) & $p$ & OR (CI 95\%) & $p$ & OR (CI 95\%) & $p$ \\
\hline \multicolumn{7}{|c|}{$\begin{array}{l}\text { Gender* } \\
\text { Female - reference group (coded=0) }\end{array}$} \\
\hline Male & $1.01(0.69-1.49)$ & 0.952 & $1.08(0.75-1.56)$ & 0.681 & $1.33(0.84-2.13)$ & 0.224 \\
\hline Age (years) & $1.28(0.99-1.64)$ & 0.054 & $0.93(0.73-1.19)$ & 0.573 & $1.02(0.75-1.34)$ & 0.900 \\
\hline \multicolumn{7}{|c|}{$\begin{array}{l}\text { Education level* } \\
\text { Low }- \text { reference group (coded=0) }\end{array}$} \\
\hline Medium & $1.76(1.13-2.74)$ & 0.013 & $1.17(0.79-1.74)$ & 0.430 & $1.22(0.75-1.98)$ & 0.421 \\
\hline High & $2.47(1.55-3.95)$ & $<0.001$ & $1.18(0.77-1.80)$ & 0.454 & $0.99(0.58-1.69)$ & 0.970 \\
\hline \multicolumn{7}{|c|}{$\begin{array}{l}\text { Body mass index } \\
\text { Normal - reference group (coded=0) }\end{array}$} \\
\hline Overweight & $0.84(0.59-1.19)$ & 0.337 & $1.01(0.72-1.40)$ & 0.972 & $2.22(1.46-3.36)$ & $<0.001$ \\
\hline Obesity & $0.89(0.56-1.42)$ & 0.625 & $0.91(0.58-1.43)$ & 0.684 & $1.48(0.84-2.60)$ & 0.178 \\
\hline
\end{tabular}

*FOP: Front-of-Pack; OR: Odds Ratio; CI: Confidence Intervals. Education level: Low- elementary school; medium - middle-school, highschool or technical career; high- bachelor’s or graduate degree. Age: continuous variable; BMI measurement followed the World Health Organization Criteria: normal weight-18.5-24.9 kg/m2; overweight: 25-29.9 kg/m2; obesity >30kg/m2 (World Health Organization 1995). Multiple logistic regression models were adjusted by gender, age, education level, and BMI. Reference groups (coded=0): gender-female; Education level-low; BMI-Normal weight.

When participants were asked which form of FOP nutrition label system they preferred, the TL FOP label was preferred (41.8\%), followed by WL (34.8\%) and the least preferred was GDA (23.4\%). There were no statistically significant differences between countries.

When zooming-in into groups stratified by education level and BMI category (i.e., normal weight, overweight and obesity) we found significant differences. Most of the parents that preferred the GDA belonged to the high education level group $(29 \%, p=0.041)$, then medium education level (23.3\%), and the least proportion were from the low education level (22.6\%). No statistical differences were found between participants that preferred the TL and education level. However, the proportion of respondents that preferred warning labels were higher from the low education level group $(40.6 \%, p=0.005)$ in comparison with those with medium education level (32.5\%) and high education level (27.2\%). Regarding BMI category, statistical differences were 
found just between participants that preferred the warning labels $(p<0.001)$, as detailed in Table 3.

Table 3. Preferences of FOP nutrition labels among Latin American parents from four countries.

\begin{tabular}{|c|c|c|c|c|c|c|c|c|c|c|c|c|c|c|}
\hline & \multicolumn{5}{|c|}{ Country } & \multicolumn{4}{|c|}{ Education Level* } & \multicolumn{4}{|c|}{ BMI Category* } & \multirow{2}{*}{$\begin{array}{c}\text { Total } \\
(\%)\end{array}$} \\
\hline & 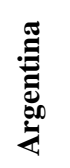 & 䒕 & 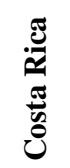 & 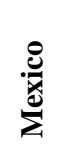 & $p$ & 登 & 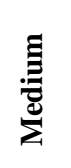 & .0ี & $p$ & 要 & 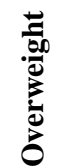 & 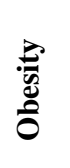 & $p$ & \\
\hline GDA (\%) & 15.7 & 20.7 & 26.7 & 25.4 & 0.535 & 22.6 & 23.3 & 29 & 0.041 & 19.9 & 23.9 & 20.5 & 0.24 & 23.4 \\
\hline TL (\%) & 50.6 & 42.1 & 38.8 & 41.7 & 0.847 & 36.8 & 44.2 & 43.8 & 0.989 & 45.2 & 40.9 & 40.2 & 0.995 & 41.8 \\
\hline $\begin{array}{l}\text { Warning } \\
\text { label (\%) }\end{array}$ & 33.7 & 37.2 & 34.5 & 32.9 & 0.184 & 40.6 & 32.5 & 27.2 & 0.005 & 34.9 & 35.2 & 20.5 & $<0.001$ & 34.8 \\
\hline
\end{tabular}

*FOP: Front-of-pack; GDA: Guideline Daily Amounts; TL: Traffic light; Education level: Low- elementary school; medium - middle-school,

high-school or technical career; high- bachelor’s or graduate degree. Body Mass Index (BMI)measurement followed the World Health

Organization Criteria: normal weight-18.5-24.9 kg/m2; overweight: $25-29.9$ kg/m2; obesity >30kg/m2 (World Health Organization 1995). A oneway comparison between countries, level of education and BMI FOP nutrition label system preference.

\section{Qualitative resullts}

From the generated codes, we developed 10 themes that afterwards were put into categories. Parents consistently expressed three main reasons to explain their preference towards a FOP nutrition label system: 1) easy to read: when the answer was oriented to the ease of use and characteristics that help to read it such as clear, fast, and easy; 2) content: responses about the information displayed, focus on the energy or calories and nutrients; and 3) attract attention, explanations centered on visual characteristics, graphics, colors and attention grabber (Table 4).

Table 4. Main reasons from the Latin American parents’ preferences of FOP* nutrition label systems

\begin{tabular}{|c|c|}
\hline Category & Codes description \\
\hline 1. Easy to read & $\begin{array}{ll}\text { - } & \text { Easy to understand and to compare within category of products } \\
\text { - } & \text { Fast and simple to use } \\
\text { - } & \text { Has large letters, visible, and colors improves skills to make } \\
\text { better choices }\end{array}$ \\
\hline 2. Content & $\begin{array}{ll}\text { - } & \text { Familiar to the labelling, has used the label before } \\
\text { - } & \text { Credibility of the information, reliable } \\
\text { - } & \text { Displayed information (i.e., energy and nutrients) }\end{array}$ \\
\hline 3. Attracts attention & $\begin{array}{ll}\text { - } & \text { The image is attractive } \\
\text { - } & \text { Color is relevant, it is easy to identify in the package } \\
\text { - } & \text { Attention grabber }\end{array}$ \\
\hline
\end{tabular}

*Front-of-Pack (FOP) nutrition label use from Argentina, Chile, Costa Rica and Mexico ( $\mathrm{n}=966)$. Categories were created from 10 themes: familiar, reliable, easy to read, easy to understand, ability to compare, content, attention grabber, fast, simple, and negative attitudes.

Respondents that preferred the GDA system consistently explained that it contained valuable information and the "content" guided their selection. 
"I understand the percentage of the daily nutrients that I need to consume and it provides information to detect the number of calories per portion; it also has information about sodium, sugar, fats and fiber, which I consider important to control in my diet." (parent, high-education level).

When TL was preferred, this was consistently due to its colors which "attract attention” and its "content."

"The colors allow one to see the information rapidly and easily." (parent, low-education level).

"For the colors, and also it delivers information on the high, medium and low levels of the nutrients." (parent, high-education level).

Participants who preferred the WL consistently explained that this format is "easy to read" and "attracts attention" since this FOP is easy to understand and has characteristics that improves the ability to make healthier choices.

" "For the displayed words that are big, and the black color clearly demonstrates what is not good." (parent, low-education level)

"Well when we do groceries, we are always running out of time, therefore almost never read the nutrition labels, but this one is short, clear and the information is big." (parent, high-education level).

\section{Discussion}

The present study assessed the usage of and preferences for three FOP nutrition label systems across four Latin American countries. Overweight parents from Argentina, Chile, Costa Rica and Mexico with low education level preferred the WL system. Other studies assessing similar FOP nutrition label systems have shown that in general, people prefer and use more a system that is easy to use and can be rapidly interpreted. (See references below.)

Parents from Mexico and Argentina reported using the FOP nutrition labels less often when purchasing food and beverage products for their children. The Mexican responses may be explained by the type of FOP labels that were in place at the time of the study. In 2016, Mexico had FOP GDA nutrition label system in place. This label began as a voluntary system relying on a nutrient profile system established by the food and beverage industry, which is currently used globally by several food and beverage corporations. Previous research had shown that this system requires mathematical skills and nutrition knowledge to calculate the energy and nutrient intake per serving, which makes it hard to understand (Stern et al. 2011; De la Cruz-Gongora et al. 2017). Consistent with past research, we found that the FOP GDA label is less often used by people to make decisions at the time of purchase compared to the other two systems (Nieto et al. 2019; Vargas-Meza et al. 2019; Talati et al. 2019). These results suggest, that simpler versions of FOP labels could reach a higher number of consumers, especially in low-and middle-income countries, where populations with low literacy levels still prevail. In contrast, the Argentinean results might be explained because of it less balanced sample (more participants with low and middle education). We found that overall parents with a higher education reported using FOP nutrition labels more often. 
The aggregated results from the 966 participants showed an overall preference on the TL system with no differences across countries. Aligned with our study, Talati et al. (2019) conducted an experimental study across 12 countries, where results favored the TL system and the outcomes were not bound to the country in spite of using other methods. Despite that the TL has been constantly preferred in previous research, the system has a limitation, it has been demonstrated that the yellow color creates confusion, especially among foods and beverages displaying a combination of colors (De la Cruz-Gongora et al. 2017; Vargas-Meza et al. 2019; Khandpur et al. 2018).

Food and nutrition policies that attempt to tackle obesity have a high risk of failure when vulnerable populations are not protected (Swinburn et al. 2019). Given our finding that the most vulnerable parents (i.e., those with low education and overweight) preferred the WL system, this system could be considered as the optimal option for governments that are seeking to implement robust strategies to tackle obesity. Parents from Argentina, Chile, Costa Rica, and Mexico that preferred the WL system explained that it is easy to read and attracts attention. Similar results have been found in other studies conducted in the Latin American region. A randomized study with 1,607 Brazilian adults highlighted a high acceptability of the warning labels due to a better visibility compared to other systems (Khandpur et al. 2018). In Chile, an article that describes the policy process of the warning label implementation also emphasized that this system proves to have high visualization and high understanding (Reyes et al. 2019). Research on Mexican, Latinos, and White populations also demonstrated that the warning label is an effective system, accepted and understood by low-and-middle income consumers (Vargas-Meza et al. 2019; Nieto et al. 2019; Arrúa et al. 2017). All these studies also found that despite the level of education or gender, the warning labels also have the ability to shift purchase decisions into healthier options by discouraging purchase of foods displaying the "excess" label.

Despite the published evidence on the effectiveness and high acceptability of the warning labels, food and beverage transnational corporations still oppose this FOP label system. Therefore, evidence on how people use, interpret and prefer a FOP nutrition label system needs to be accompanied by political will and strong support by civil society and organizations that aim to protect public health. Further research should be done to understand the political will and policymaking process in the area. Other research should further investigate the different nutrient criteria to improve the robustness of FOP nutrition label policies.

This paper is particularly relevant to sum evidence for advocating for better and stronger food labeling systems worldwide, but particularly in Latin American countries; it also provides further input into current work Codex Alimentarius is doing on FOP nutrition label systems.

\section{Limitations of the study}

The study used a self-administered questionnaire, which could under-estimate participant's weight, therefore their BMI category, and over-estimate label use (Cowburn and Stockley 2005). Although we used a sample of four countries, the study does not involve all the countries of the Latin American region; therefore, it is not a representative study from Latin America. In addition, we used a convenience sample of parents; therefore, the participants from the study cannot be considered a representative sample. However, the variety and large size of the sample 
allowed us to find statistically significant results among people with low education and overweight, common characteristics in many Latin American populations.

\section{Conclusions}

Among the three FOP systems we examined, the warning label system was preferred by the most vulnerable parents (i.e., low education level and overweight) across four Latin American countries. With the stability of findings across countries and with previous evidence for other research, this study suggests that an easily understandable, readable and acceptable label may facilitate usage by vulnerable groups to make healthy food purchases. Warning labels appear to be the most accepted format in Latin America since it complies with all the characteristics previously described. Besides, currently six countries in the region have adopted the FOP warning label system. Increasingly, countries are using FOP nutrition label systems as one costeffective obesity prevention strategy. This research suggests that serious attention be given to choosing the warning label system.

\section{Acknowledgements}

The authors greatly appreciate Sara Hendery and Vivica Kraak for their help in proofreading the manuscript, Alejandra Castillo for her support on supervising and cleaning the databases, and Verónica García for her input into the qualitative analysis.

\section{Competing interests}

The authors declare that they have no competing interests.

\section{Authors' contributions}

S.R.G.P led the study conception and design, analysis, data collection and writing of the draft manuscript. The co-authors contributed as follows: conceptualization, A.C., L.T., and S.B.; methodology, A.C. and S.B.; statistical analysis, J.A.; validation, A.C.; editing, J.A., A.C., L.T., L.A., A.M., M.S., L.B., S.B.; All authors read and approved the final manuscript.

\section{Funding}

This research was funded by United Nations Children's Fund (UNICEF) through a grant \#43188882 obtained in 2016. 


\section{References}

Anastasiou, K., M. Miller, and K. Dickinson. 2019. "The relationship between food label use and dietary intake in adults: A systematic review." Appetite 138: 280-291. https://doi.org/10.1016/j.appet.2019.03.025.

Arrúa, A., L. Machín, and MR Curutchet, et al. 2017. "Warnings as a directive front-of-pack nutrition labelling scheme: comparison with the Guideline Daily Amount and traffic-light systems." Public Health Nutrition 20 (13): 2308-2317. https://doi.org/10.1017/S1368980017000866.

Campos, S., J. Doxey, and D. Hammond. 2011. "Nutrition labels on pre-packaged foods: a systematic review." Public Health Nutr 14 (8): 1496-506. https://doi.org/10.1017/s1368980010003290.

Carter, N., D. Bryant-Lukosius, A. DiCenso, et al. 2014. "The use of triangulation in qualitative research." Oncol Nurs Forum 41 (5): 5457. https://doi.org/10.1188/14.ONF.545-547.

Cowburn, G., and L. Stockley. 2005. "Consumer understanding and use of nutrition labelling: a systematic review." Public Health Nutr 8 (1): 21-8. https://doi.org/10.1079/phn2005666.

De la Cruz-Gongora, V., P. Torres, A. Contreras-Manzano, et al. 2017. "Understanding and acceptability by Hispanic consumers of four front-of-pack food labels." Int J Behav Nutr Phys Act 14 (1): 28. https://doi.org/10.1186/s12966-017-0482-2.

De la Cruz-Gongora, V., S. Villalpando, G. Rodriguez-Oliveros, et al. 2012. "Use and understanding of the nutrition information panel of pre-packaged foods in a sample of Mexican consumers." Salud Publica Mex 54 (2): 158-66. https://doi.org/10.1590/s003636342012000200012.

Drewnowski, A., and C.D. Rehm. 2013. "Energy intakes of US children and adults by food purchase location and by specific food source." Nutrition Journal 12 (1): 59. https://doi.org/10.1186/1475-2891-12-59.

Ezzati M, and E. Riboli. 2013. "Behavioral and Dietary Risk Factors for Noncommunicable Diseases." New England Journal of Medicine 369 (10): 954-964. https://doi.org/10.1056/NEJMra1203528.

Food and Agricultural Organization. 2019. Food Labeling. http://www.fao.org/food-labelling/en/

Graña M. 2018. New Front-of-Package Labeling Regulation in Uruguay. https://galalaw.com/Publication/GazetteArticle?id=1268\&utm_source=Mondaq\&utm_me dium=syndication\&utm_campaign=View-Original.

Jiwani S. S, Carrillo-Larco R. M, Hernandez-Vasquez A, T, et al. 2019. "The shift of obesity burden by socioeconomic status between 1998 and 2017 in Latin America and the Caribbean: a cross-sectional series study." Lancet Glob Health 7 (12): e1644-e1654. https://doi.org/10.1016/s2214-109x(19)30421-8.

Khandpur, N., PdM Sato, LA Mais, et al. 2018. "Are Front-of-Package Warning Labels More Effective at Communicating Nutrition Information than Traffic-Light Labels? A Randomized Controlled Experiment in a Brazilian Sample." Nutrients 10 (6): 688. https://doi.org/10.3390/nu10060688.

Kline, L, J Jones-Smith, J Jaime Miranda, et al. 2017. "A research agenda to guide progress on childhood obesity prevention in Latin America." Obes Rev 18, (2): 19-27. https://doi.org/10.1111/obr.12572.

Massey, A. 1999. "Methodological triangulation, or how to get lost without being found out." Studies in Educational Ethnography 2: 183-197. 
Michail N. 2019, "Peru: Nutrition warning labels become mandatory," https://www.foodnavigator-latam.com/Article/2019/06/17/Peru-Nutrition-warning-labelsbecome-mandatory.

Ministry of Health Chile. 2015. Law 20.606. https://www.minsal.cl/wpcontent/uploads/2019/08/EVALUACION-LEY-DE-ALIMENTOS_julio-2019_02.pdf

Ministry of Health Mexico. 2014. LINEAMIENTOS por los que se dan a conocer los criterios nutrimentales y de publicidad que deberán observar los anunciantes de alimentos y bebidas no alcohólicas para publicitar sus productos en televisión abierta y restringida, así como en salas de exhibición cinematográfica, conforme a lo dispuesto en los artículos 22 Bis, 79, fracción X y 86, fracción VI, del Reglamento de la Ley General de Salud en Materia de Publicidad. http://www.dof.gob.mx/nota_detalle.php?codigo=5340694\&fecha=15/04/2014

Ministry of Public Health Ecuador. 2013. Reglamento de Etiquetado de Alimentos Procesados para el Consumo Humano. (Ecuador). https://www.controlsanitario.gob.ec/wpcontent/uploads/downloads/2014/08/REGLAMENTO-SANITARIO-DEETIQUETADO-DE-ALIMENTOS-PROCESADOS-PARA-EL-CONSUMOHUMANO-junio-2014.pdf.

Ng M., T. Fleming, M. Robinson, B. Thomson, et al. 2014. "Global, regional, and national prevalence of overweight and obesity in children and adults during 1980-2013: a systematic analysis for the Global Burden of Disease Study 2013." Lancet 384 (9945): 766-81. https://doi.org/10.1016/S0140-6736(14)60460-8. https://www.ncbi.nlm.nih.gov/pubmed/24880830.

Nieto, C., A. Jáuregui, A. Contreras-Manzano, et al. 2019. "Understanding and use of food labeling systems among Whites and Latinos in the United States and among Mexicans: Results from the International Food Policy Study, 2017." International Journal of Behavioral Nutrition and Physical Activity 16 (1): 87. https://doi.org/10.1186/s12966019-0842-1. https://doi.org/10.1186/s12966-019-0842-1.

Pan American Health Organization. 2019. Ultra-processed Food and Drink Products in Latin America: Sales, sources, nutrient profiles, and policy implications. http://iris.paho.org/xmlui/handle/123456789/51094.

Popkin, B., and T. Reardon. 2018. "Obesity and the food system transformation in Latin America." Obesity Reviews 19: 1028-1064. https://doi.org/10.1111/obr.12694.

Poti, J. M., M. M. Slining, and B. M. Popkin. 2014. "Where are kids getting their empty calories? Stores, schools, and fast-food restaurants each played an important role in empty calorie intake among US children during 2009-2010." J Acad Nutr Diet 114 (6): 908-17. https://doi.org/10.1016/j.jand.2013.08.012.

Reyes, M., M.L. Garmendia, S. Olivares, et al. 2019. "Development of the Chilean front-ofpackage food warning label." BMC Public Health 19 (1): 906. https://doi.org/10.1186/s12889-019-7118-1. https://doi.org/10.1186/s12889-019-7118-1.

Rivera, JA, TG de Cossio, LS Pedraza, et al. 2014. "Childhood and adolescent overweight and obesity in Latin America: a systematic review." Lancet 2 (4): 321-332. https://linkinghub.elsevier.com/retrieve/pii/S2213-8587(13)70173-6.

Ronit, K., and J. Dejgård Jensen. 2014. "Obesity and industry self-regulation of food and beverage marketing: a literature review." European Journal of Clinical Nutrition 68 (7): 753-759. https://doi.org/ 10.1038/ejcn.2014.60 
Shamah-Levy, T. L. Cuevas-Nasu, E. B. Gaona-Pineda, et al. 2016. Encuesta Nacional de Salud y Nutrición de Medio camino 2016. (Instituto Nacional de Salud Pública). https://www.gob.mx/cms/uploads/attachment/file/209093/ENSANUT.pdf Shangguan, S., A. Afshin, M. Shulkin, et al. 2019. "A Meta-Analysis of Food Labeling Effects on Consumer Diet Behaviors and Industry Practices." Am J Prev Med 56 (2): 300-314. https://doi.org/10.1016/j.amepre.2018.09.024.

Sherman A. 2019, "Mexico votes to strengthen food and beverage warning labels." https:/www.foodnavigator-latam.com/Article/2019/10/28/Mexico-votes-to-strengthenfood-and-beverage-warning-labels.

Stern D., L. Tolentino, and S. Barquera. 2011. "Revisión del etiquetado frontal: análisis de las Guías Diarias de Alimentación (GDA) y su comprensión por estudiantes de nutrición en México." Instituto Nacional de Salud Pública. http://pliopencms05.salud.gob.mx:8080/BasesCDI/Archivos/EstudioseInvestigaciones/re visionEtiquetadoFrontal.pdf

Swinburn, B. A., V. I. Kraak, S. Allender, et al. 2019. "The Global Syndemic of Obesity, Undernutrition, and Climate Change: The Lancet Commission report." Lancet 393 (10173): 791-846. https://doi.org/10.1016/s0140-6736(18)32822-8.

Talati, Z., M. Egnell, S. Hercberg, et al. 2019. "Food Choice Under Five Front-of-Package Nutrition Label Conditions: An Experimental Study Across 12 Countries." American Journal of Public Health 109 (12): 1770-1775. https://doi.org/10.2105/ajph.2019.305319.

Vargas-Meza, J., A. Jauregui, S. Pacheco-Miranda, et al. 2019. "Front-of-pack nutritional labels: Understanding by low- and middle-income Mexican consumers." PLoS One 14 (11): e0225268. https://doi.org/10.1371/journal.pone.0225268.

World Health Organization. 1995. Physical status: the use and interpretation of anthropometry. Report of a WHO Expert Committee. World Health Organization (Geneva, Switzerland). https://apps.who.int/iris/bitstream/handle/10665/37003/WHO_TRS_854.pdf;jsessionid=E 6FC6BEA750E45C871CE88A7B924A9B4?sequence=1.

World Health Organization. 2013. Action Plan for the Prevention and Control of Noncommunicable Diseases 2013-2020. (Geneva, Switzerland, WHO,). http://apps.who.int/iris/bitstream/10665/94384/1/9789241506236_eng.pdf?ua=1.

World Health Organization. 2018. "Obesity and overweight - Fact Sheet." https://www.who.int/news-room/fact-sheets/detail/obesity-and-overweight.

World Health Organization. 2019a. Why does childhood overweight and obesity matter? https://www.who.int/dietphysicalactivity/childhood_consequences/en/

World Health Organization. 2019b. Guiding principles and framework manual for front-of-pack labelling for promoting healthy diet. https://www.who.int/nutrition/publications/policies/guidingprinciples-labellingpromoting-healthydiet.pdf?ua=1 